\title{
An efficient and information-rich biochemical method design for fragment library screening on ion channels
}

\author{
Andrew J. Thompson ${ }^{1}$, Mark H.P. Verheij ${ }^{2}$, Rob Leurs ${ }^{2}$, Iwan J.P. De Esch ${ }^{2}$, and Sarah C.R. Lummis ${ }^{1}$ \\ ${ }^{1}$ Department of Biochemistry, University of Cambridge, Cambridge, UK and ${ }^{2}$ Medicinal Chemistry, VU University \\ Amsterdam, Amsterdam, The Netherlands
}

BioTechniques 49:822-829 (November 2010) doi 10.2144/000113538

Keywords: fragment; library; 5- $\mathrm{HT}_{3}$; cys-loop; ligand; high-throughput; screen; therapeutic

Drug discovery requires a simple, rapid, and cost-effective method for the early identification of novel leads and elimination of poor candidates. Here we present an experimental design that fulfils these criteria, using a ligandgated ion channel expressed in a mammalian cell line, whose function can be probed using a voltage-sensitive dye. The experimental design is novel, as it uses the same screen to identify hit fragments and to characterize them as agonists or antagonists. The results were independently validated using radioligand binding, although the new technique has several advantages over radioligand methods. A number of novel high-affinity ligands were found. The method is broadly applicable to a wide range of receptor types including ligand-gated ion channels (LGICs), voltage-gated ion channels (VGICs), and G protein-coupled receptors (GPCRs), all of which are important drug targets.

Fragment-based drug discovery (FBDD) is an efficient and rational drug discovery approach that is becoming widely adopted by industry and academia (1-5). An essential aspect of FBDD is the identification of low molecular weight fragments that bind to their biological target and can be efficiently transformed into higher molecular weight drug candidates (6). Biophysical fragment screening approaches such as NMR and surface plasmon resonance (SPR) are frequently used to initiate FBDD projects, but biochemical assays are also needed, especially when searching for targets such as enzyme inhibitors $(7,8)$. Biochemical fragment screening assays have also been described for transmembrane receptors such as G protein-coupled receptors (GPCRs) and ligand-gated ion channels (LGICs) $(9,10)$. Both of these drug target classes can be modulated by compounds that activate (agonists) or inhibit (antagonists) the receptor, but no biochemical or radioligand fragment screening protocols have been described that detect both these actions in a single screen. This is critical, as even within the same chemical series, small structural differences of ligands can change their functional response.

LGICs include nicotinic acetylcholine (nACh), $\gamma$-aminobutyric acid $(\mathrm{GABA})_{\mathrm{A}}$, glycine, and 5-hydroxytryptamine ${ }_{3}\left(5-\mathrm{HT}_{3}\right)$ receptors, all of which are important therapeutic targets (11-13). The majority of known LGIC drugs act at the orthosteric binding site, but there are also other binding sites that can be valuable targets for drugs (14). Both radioligand binding assays and in silico methods tend to concentrate on the orthosteric binding site, thereby potentially missing activity at important target sites elsewhere in the receptor; a test of candidate fragments on functional receptors allows activity at all binding sites to be monitored. Here we describe a novel method design for screening a structurally diverse set of 1116 fragments using $5-\mathrm{HT}_{3}$ receptors stably expressed in HEK293 cells. With a single screen, the method identifies active compounds and whether they are agonists and antagonists; a second similar screen determines their potency. The approach is scalable, simple, and fast; uses commercially available components; and is generally applicable. As such, it constitutes a unique and informationrich biochemical screen that can rapidly generate key data for drug discovery.

\section{Materials and methods}

Cell preparation

HEK293 cells were transfected with the human $5-\mathrm{HT}_{3} \mathrm{~A}$ subunit (RCSB Protein Data Bank ID no.P46098; www.pdb.org) in pcDNA3.1 (Invitrogen, Paisley, UK). Stably expressing cells were clonally selected with the addition of G-418 (Sigma-Aldrich, Dorset, UK). Cells were routinely grown in $90-\mathrm{mm}$ dishes containing DMEM/Glutamax (Gibco, Invitrogen) until $90 \%$ confluent and transferred to black 96-well plates (Greiner Bio-One, Stonehouse, UK) treated with $0.01 \%$ Cultrex poly-L-lysine (Trevigen, Gaithersburg, MD, USA). To maximize fluorescence, cells were used when densities had reached $100 \%$ (i.e., cells were tightly packed), which was usually 2-3 days later.

Dye loading

HEK293 cells stably expressing the human $5-\mathrm{HT}_{3}$ receptor were washed twice with buffer $(115 \mathrm{mM} \mathrm{NaCl}, 1 \mathrm{mM} \mathrm{KCl}, 1 \mathrm{mM}$ $\mathrm{MgCl}_{2}, 1 \mathrm{mM} \mathrm{CaCl}, 10 \mathrm{mM}$ HEPES, $10 \mathrm{mM}$ D-glucose, $\mathrm{pH} 7.4$ ) and incubated for $60 \mathrm{~min}$ with $100 \mu \mathrm{L}$ fluorescent membrane potential (FMP) dye dissolved in the same buffer (FLIPR Membrane Potential Assay kit; Molecular Devices, Wokingham, UK). Test compounds were added directly to these cells without removing the dye buffer.

Compound preparation

Two compound plates were constructed for each screen; one containing novel 
A

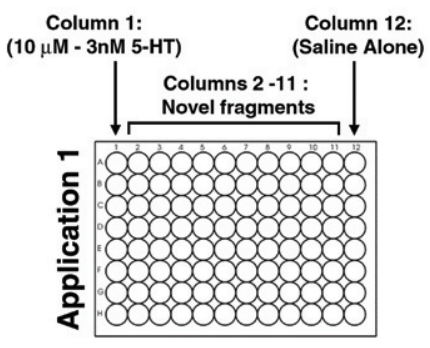

B

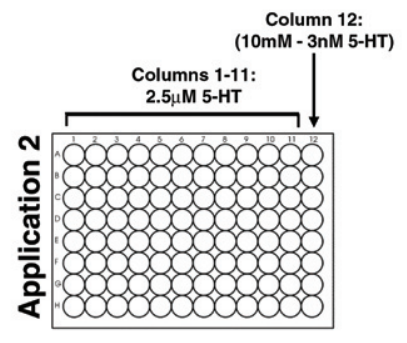

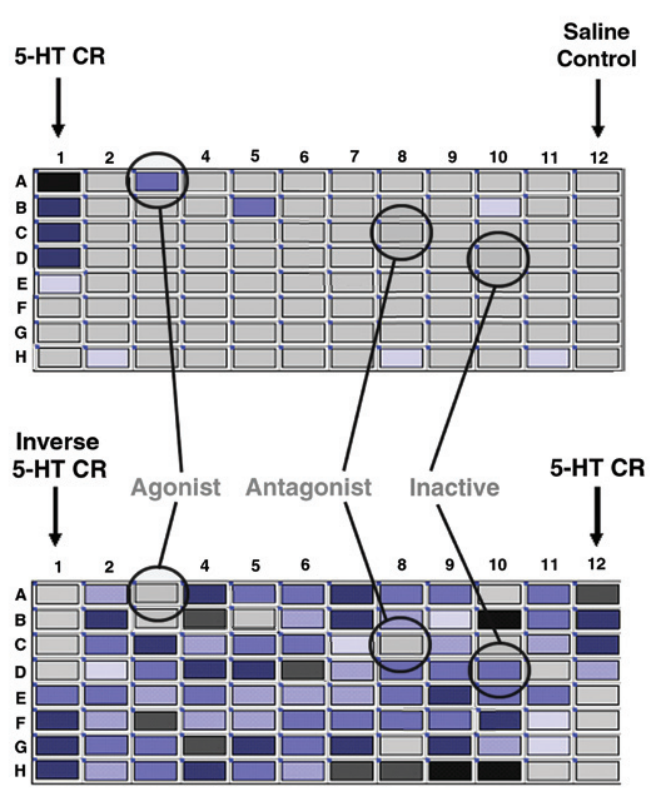

C

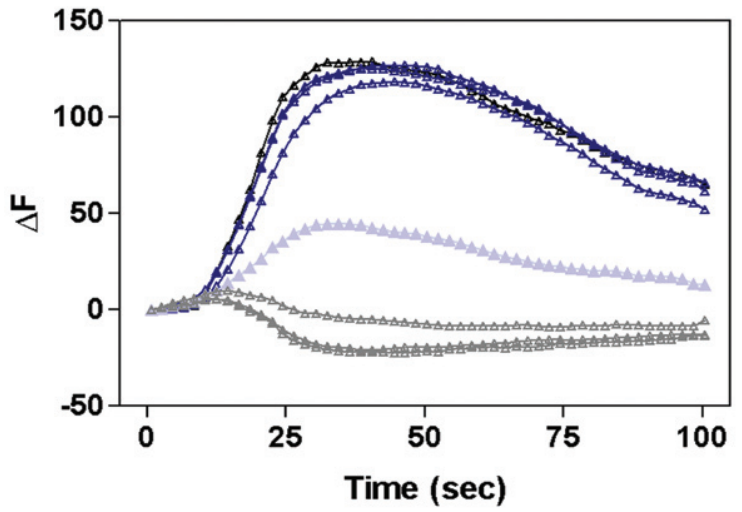

SCALE

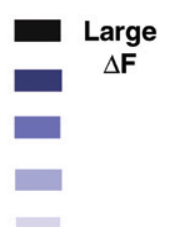

Small

$\Delta \mathbf{F}$

Figure 1. Plate composition. (A) As controls, column 1 of application 1 is a serial dilution of a known agonist, and column 12 is saline alone. Compounds from the fragment library are added to each of the remaining wells. Column 12 of application 2 is a serial dilution of a known agonist, the response to which can be compared with column 1 of application 1. (B) Fluorescent response of typical agonist, antagonist, and inactive compound fragments. Colors are assigned by the software, ranging from the largest fluorescence change (black) to the smallest (grey). In this example, the 5-HT concentration-response in column 1 of application 1 and column 12 of application 2 are similar, showing that fluorescent responses were consistent throughout the full 40-min experiment. In column 1 of application 2, an inverse 5-HT concentration response is seen because of desensitization at high concentrations of agonist during the first application. (C) Shown is the 5-HT concentration response data plotted as a change in fluorescence over time. The peak values from these are same as those in column 1 of panel B. CR, concentration response.

fragments and one containing 5-HT (Figure 1). In the first plate, compounds from the fragment library were added to columns $2-11$ at a concentration of 300 $\mu \mathrm{M}$ (Figure 1). Column 1 was a 5-HT concentration-response (3-fold serial dilutions from $30 \mu \mathrm{M}$ to $10 \mathrm{nM}$ over 8 rows), and column 12 was filled with buffer alone. All concentrations described in the methods are the concentrations in the compound plate. Those described in the results and figure legends are final concen- trations in the cells. The 5-HT concentration response ensured that the cells gave consistent readings at the beginning of each experiment and between plates. Concentration dependence is a more robust control than using a single concentration, as it provides a highly accurate method of confirming consistent 5-HT responses and cell viability (a potentially serious problem due to the high compound concentrations commonly used with fragment screening). The saline blank ensured that an appli-

\section{Having Difficulty Amplifying GC-Rich Targets?}

Introducing CleanAmp ${ }^{\text {TM }}$ 7-Deaza-dGTP as an innovative Hot Start PCR solution for amplification of problematic GC-rich sequences.

Experience a Versatile Solution

Achieve Enhanced Specificity

Commercialize for Less with Reasonable Licensing Options

See Page 841 for Further Details! 
cation of buffer alone did not alter the baseline fluorescence and allowed a final 5-HT concentration-response to be performed at the end of the second plate. Before commencing our experiments, these plates were assessed for compound fluorescence, and no significant levels were found. Quenching was not measured as the FMP dye used in this study is known to be less affected than other fluorescent probes, and this was confirmed by the low numbers of false positives; if necessary, quenching can detected using artifact plates as suggested by Shapiro et al. $(15,16)$.

In the second compound plate, $10 \mu \mathrm{M} 5-\mathrm{HT}$ was added to columns 1-11, and column 12 was a $5-\mathrm{HT}$ concentrationresponse (3-fold serial dilutions from $30 \mu \mathrm{M}$ to $10 \mathrm{nM}$ over 8 rows). The concentration-response curves from column 1 of plate 1 and column 12 of plate 2 were compared to ensure that cell viability was maintained throughout the two assays. Since $50 \mu \mathrm{L}$ $10 \mu \mathrm{M} 5$-HT was added to $150 \mu \mathrm{L}(100 \mu \mathrm{L}$ dye and $50 \mu \mathrm{L}$ novel compound from application 1), the final 5-HT concentration was $4 \times$ lower (i.e., $2.5 \mu \mathrm{M}$ ) than the starting concentration. This concentration of 5-HT was ideal as it maximized the amplitude of the observed 5-HT response, but was low enough for $\mathrm{IC}_{50}$ values to be derived (Figures 1 and 2).

Hit compounds were further assayed to confirm their activity and to obtain an accurate measure of their potency. For this experiment, each compound was tested at a range of concentrations (Figure 2, C and D, columns 2-11). 5-HT and saline controls were as described above. Antagonists yielded fluorescence measurements that could be plotted as concentration-inhibition curves (Equation 1; Figure 2D, right of panel). The 5-HT control had a concentration response relationship that was reversed in the second application because of receptor desensitization. When the

\section{BioTechnioues}

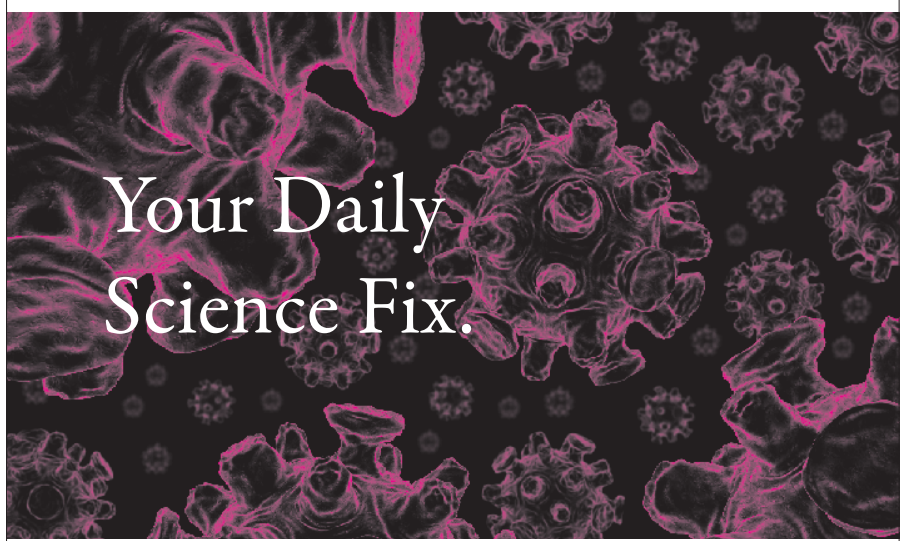

As one of the most comprehensive information sources for biological researchers, BioTechniques' daily newsletter keeps you informed of the most recent developments, products, and industry news in the life sciences. We compile the latest information from the past 24 hours in one convenient source.

Not a subscriber? Sign up for free at: www.BioTechniques.com/newsletters curves from the first and second screens were plotted, the two curves crossed (Equation 1; Figure 2C, right of panel).

\section{Fluorometric assays}

Dye-loaded cells were transferred to a FlexStation II (Molecular Devices). Fluorescence was recorded every $2 \mathrm{~s}$ for $20 \mathrm{~s}$, to provide a baseline level, after which the compounds were added, and the change in fluorescence was recorded every $2 s$ for a further $80 s$ (Figure 1). Fluorescence levels were measured using Softmax Pro v4.3 (Molecular Devices) and exported to Microsoft Excel 2002 SP3 (Redmond, WA, USA) for analysis.

\section{Categorizing agonists, antagonists, and non-hits}

Compounds were scored as agonists, antagonists, or inactive (Figure 1). Agonists were defined as compounds that displayed an increase in fluorescence of at least $50 \%$ of that obtained with maximum $(30 \mu \mathrm{M}) 5$ - $\mathrm{HT}$ and $<30 \%$ maximum 5 -HT activity in the second application. Antagonists caused little $(<20 \%)$ or no change in fluorescence relative to the maximum 5 -HT response in the first application, but inhibited the 5-HT evoked response during the second application. The thresholds were derived from reference compound data; at these values known agonists and antagonists are clear hits, and the number of false hits and lower affinity compounds are minimal. The stringency of the screen can be changed by altering these thresholds and would depend on the system being studied and the number of false hits that are considered acceptable. If a compound was identified in two of three replicate plates, it was considered a hit and assessed further by concentration response and radioligand binding (Figure 2).

\section{Analysis and curve fitting}

Analysis and curve fitting was performed using Prism v3.02 (GraphPad Software, San Diego, CA, USA). Concentration dependence data was normalized to the maximum fluorescence elicited. The mean and SEM for at least three independent runs were plotted against agonist or antagonist concentration and iteratively fitted to the following equation,

$$
I_{A}=I_{\min }+\frac{I_{\max }-I_{\min }}{1+10^{n_{H}\left(\log A_{50}-\log A\right)}}
$$

where $A$ is the concentration of ligand present, $I_{A}$ is the current in the presence of ligand concentration $A, I_{\min }$ is the current when $A=0, I_{\max }$ is the current when $A=\infty, A_{50}$ is the concentration of $A$ that evokes a current equal to $\left(I_{\max }+I_{\min }\right) / 2$, and $n_{H}$ is the Hill coefficient. $K_{\mathrm{b}}$ was estimated from $\mathrm{IC}_{50}$ values using the Cheng-Prusoff equation with the modification by Leff and Dougall (17),

$$
K_{\mathrm{b}}=\frac{I C_{50}}{\left(2+\left(\frac{[L]}{\left[E C_{50}\right]}\right)^{n_{H}}\right)^{\frac{1}{n_{H}}}-1}
$$

[Eq. 2]

where $K_{\mathrm{b}}$ is the dissociation constant of the competing drug, $I C_{50}$ is the concentration of antagonist required to half the maximal 
response, $[L]$ is the agonist concentration, $\left[E C_{50}\right]$ is the agonist $E C_{50}$, and $n_{H}$ is the Hill slope of the agonist.

\section{Radioligand binding}

Transfected HEK293 cells were scraped into $1 \mathrm{~mL}$ ice-cold HEPES buffer $(10 \mathrm{mM}$, $\mathrm{pH} 7.4$ and frozen). After thawing, they were washed with HEPES buffer and homogenized; then $50 \mu \mathrm{g}$ cell membranes were incubated in $0.5 \mathrm{~mL}$ HEPES buffer containing $1 \mathrm{nM}\left[{ }^{3} \mathrm{H}\right]$ granisetron $\left(\sim K_{\mathrm{d}}\right)$. Competition binding (8-point) was performed on at least three separate plates of transfected cells. Nonspecific binding was determined using $1 \mathrm{mM}$ quipazine. Reactions were incubated for $\geq 12 \mathrm{~h}$ at $4^{\circ} \mathrm{C}$, to allow compounds with slow kinetics to equilibrate. Incubations were terminated by vacuum filtration using a Brandel cell harvester (Alpha Biotech, London, $\mathrm{UK}$ ) onto GF/B filters presoaked in $0.3 \%$ polyethyleneimine. Radioactivity was determined by scintillation counting using a Beckman BCLS6500 (Beckman Coulter, Fullerton, California, USA). Data were fit according to the equation

$$
B_{L}=B_{\min }+\frac{B_{\max }-B_{\min }}{1+10^{n_{H}\left(\log L_{50}-\log L\right)}},
$$

[Eq. 3]

where $L$ is the concentration of ligand present, $B_{L}$ is the binding in the presence of ligand concentration $L, B_{\min }$ is the binding when $L=0, B_{\max }$ is the binding when $L$ $=\infty, L_{50}$ is the concentration of $L$ that gives a binding equal to $\left(B_{\text {max }}+B_{\text {min }}\right) / 2$, and $n_{H}$ is the Hill coefficient. $K_{\mathrm{i}}$ values were estimated from $I C_{50}$ values using the Cheng-Prusoff Equation (18),

$$
K_{\mathrm{i}}=\frac{I C_{50}}{1+\frac{[L]}{K_{\mathrm{d}}}},
$$

where $K_{\mathrm{i}}$ is the equilibrium dissociation constant for binding of the unlabeled antagonist, $I C_{50}$ is the concentration of antagonist that blocks half the specific binding, $[L]$ is the free concentration of radioligand, and $K_{\mathrm{d}}$ is the equilibrium dissociation constant of the radioligand.

\section{Calculating fragment diversity}

The compound collection obeys standard 1-D fragment library rules, including the number of heavy atoms $<22, \log \mathrm{P}<3$, the number of $\mathrm{H}$-bond donors and $\mathrm{H}$-bond acceptors $<3$, and the number of rotatable bonds $<5$ (19). Gaussian distributions were seen for all parameters except $\log P$, where most compounds were found in the ranges of 2-3 (43\%) and 1-2 (33\%). Structural diversity of the fragment library was shown by the Scaffold Classification Approach (SCA) (20). For all fragments in the library, the cyclicity was calculated as a descriptor of cyclic and acyclic substructures, meaning that cyclicity is maximal $(=1)$ if all atoms of a fragment are contained within the system of ring structures that are defined as the scaffold. The more atoms that are part of the acyclic substituents that are attached to the scaffold, the lower the cyclicity. In addition, the complexity was calculated as a descriptor of the size and shape of the scaffold, taking into account the smallest set of smallest rings, the number of heavy atoms, the number of bonds between the heavy atoms, and the sum of heavy atoms atomic number. Calculations were performed on an Intel Core 2 Quad CPU 2.4 GHz, with 4 GB RAM running Molecular Operating Environment (MOE, version 2008.10; Chemical Computing Group, Montreal, Canada) and sca.svl, a dedicated SVL script (http://svl.chemcomp.com). The set is commercially available via IOTA Pharmaceuticals (Cambridge, UK).

\section{Results and discussion}

FBDD is an efficient approach for identifying low molecular weight drug candidates, but the screening methods do not usually determine whether a compound is an agonist or antagonist (1-10). We describe a novel method design for identifying agonist and antagonists from a structurally diverse fragment library. The method has several advantages over other methods, such as radioligand binding, as it does not depend upon the availability of a ligand with a high affinity and specific activity, it can distinguish agonists from antagonists, and, as it is not limited to interactions at a single binding site, no a priori decisions are needed regarding the site of action $(10,21)$. By using functional receptors, we were able to assess the compounds under physiological conditions. A total of 72 hits were identified from a total of 1116 fragments, and activity of these was later confirmed by demonstrating concentration-dependence (from which $\mathrm{EC}_{50}$ and $\mathrm{IC}_{50}$ estimates were calculated) and competition with a radiolabeled antagonist.

To assess compounds for both agonist and antagonist behaviors, HEK293 cells stably expressing the human $5-\mathrm{HT}_{3}$

\section{Class \\ Volumatric Plastic Labwarcel $\underset{\text { BRAND }}{\Delta}$}

- Accurate:

Class $A$ and Class $B$ versions available

- Safe \& Economical: Volumetric plasticware resists breakage, saves on replacement costs

\section{- Versatile:} Plastic options for chemical compatibility and trace analysis

BrandTech offers a full line of volumetric plastic labware \& general laboratory plastics. Available through leading lab dealers!
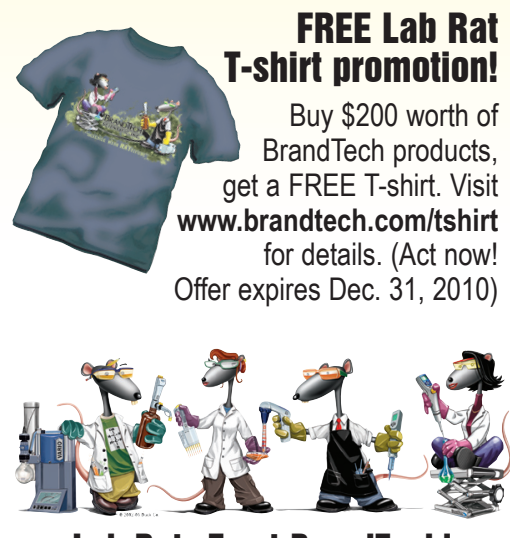

Lab Rats Trust BrandTech!

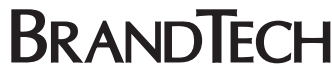
SCIENTIFIC, INC.

Toll Free (888) 522-2726 www.brandtech.com 
receptor $\left(\mathrm{B}_{\max }=2487 \pm 409 \mathrm{fmol} / \mathrm{mg}\right.$ protein, $n=7$ ) were subjected to two drug applications (Figure 1). In the first, agonist activity was assessed by adding $50 \mu \mathrm{L}$ novel library compound (to yield a final concentration of $100 \mu \mathrm{M}$ ) to each well. The volume and concentration of compound that was used ensured that only small quantities of compound were needed and only compounds with a relatively high potency were classified as hits. In the second application, $50 \mu \mathrm{L} 5-\mathrm{HT}$ (to give a final concentration of $2.5 \mu \mathrm{M}$ ) were added to the same cells. Compounds were classified as agonists if they elicited a response in the first plate, but not in the second (following activation by the first application, receptors desensitize and are unable to open again) and antagonists if they did not cause a response in either the first or second plate (the first application blocks the receptors, and they remain blocked during the second application). Compounds that did not respond to the first application, but showed a response to the second were considered inactive (receptors are unaltered by the first application and respond to the known agonist in the second). Using the same cells twice had several advantages: (i) reuse allowed for reduced material costs; (ii) the 20-min time period between the first and second applications allowed agonists and antagonists to equilibrate on the cells before 5-HT was added; and (iii) reproducibility was improved because the same cells were subjected to both applications.

The 1116 compounds were screened at least three times and scored as hits when activity was observed on at least two plates (Figure 2). This number of replicates was based on a careful assessment of the quality of hits versus the number of repeats (Figure $3 \mathrm{~A})$. Increasing the number of replicates used more compound, took more time, required more consumables, and generated a depreciating number of additional hits; those that were identified were of lower affinity. This experimental design identified a total of 72 active compounds, of which 10 were predicted to be agonists and 62 antagonists. This is an overall hit rate of $6.45 \%$, which is comparable to other FBDD and HTS studies (5,19,22-24). For these hits, a second screen was used to verify concentration-dependent activity and determine $\mathrm{EC}_{50}$ or $\mathrm{IC}_{50}$ values. This second screen was prepared and run using the same method design as the first screen, except that a range of concentrations was used for each hit. As two applications were also used in the second screen, agonist and antagonist behaviors were confirmed, and differing levels of fluorescence measured across the range of concentrations allowed curves to be constructed from which $\mathrm{EC}_{50}$ and $\mathrm{IC}_{50}$ values were calculated.

Concentration dependence was established for the 10 potential agonists selected from the initial screen, representing a $100 \%$ success rate for correctly identifying agonist hits. Of the 62 predicted antagonists from the first screen, 61 displayed concentration-dependent antagonist behavior, and 9 of these also had agonist activity at higher concentrations. Therefore, $98 \%$ of antagonists were correctly identified in the initial hit screen (i.e., there was only one false positive), with an overall success rate of $99 \%$ for correctly selecting hits irrespective of their agonist or antagonist behavior.

In addition to showing concentration dependence, hits were further validated using competition radioligand binding. Of those found to inhibit the $5-\mathrm{HT}_{3}$ response, 90\% (65/72) also displaced specific binding of the high affinity $5-\mathrm{HT}_{3}$ competitive antagonist $\left[{ }^{3} \mathrm{H}\right]$ granisetron $\left(\mathrm{p} K_{\mathrm{d}}=9.04 \pm 0.05, n=7\right)$. For these compounds, $\mathrm{p} K_{\mathrm{i}}$ estimates were calculated (Equation 4) and compared with $\mathrm{p} K_{\mathrm{b}}$ estimates from the fluorometric assay

\title{
\& mōtōnomic Redefining pipetting
} with your technique in mind.

\author{
Introducing pipetman m \\ the new ergonomic motorized pipette from Gilson \\ and the latest evolution of PIPETMAN P-the \\ world standard for over 35 years. The innovative \\ PIPETMAN M boasts multiple pipetting modes, \\ variable piston speeds, and virtually zero pipetting \\ forces! All for less than you would expect to pay \\ for a motorized pipette.
}

As simple as PIPETMAN ${ }^{\circ}$... just the way you like it.

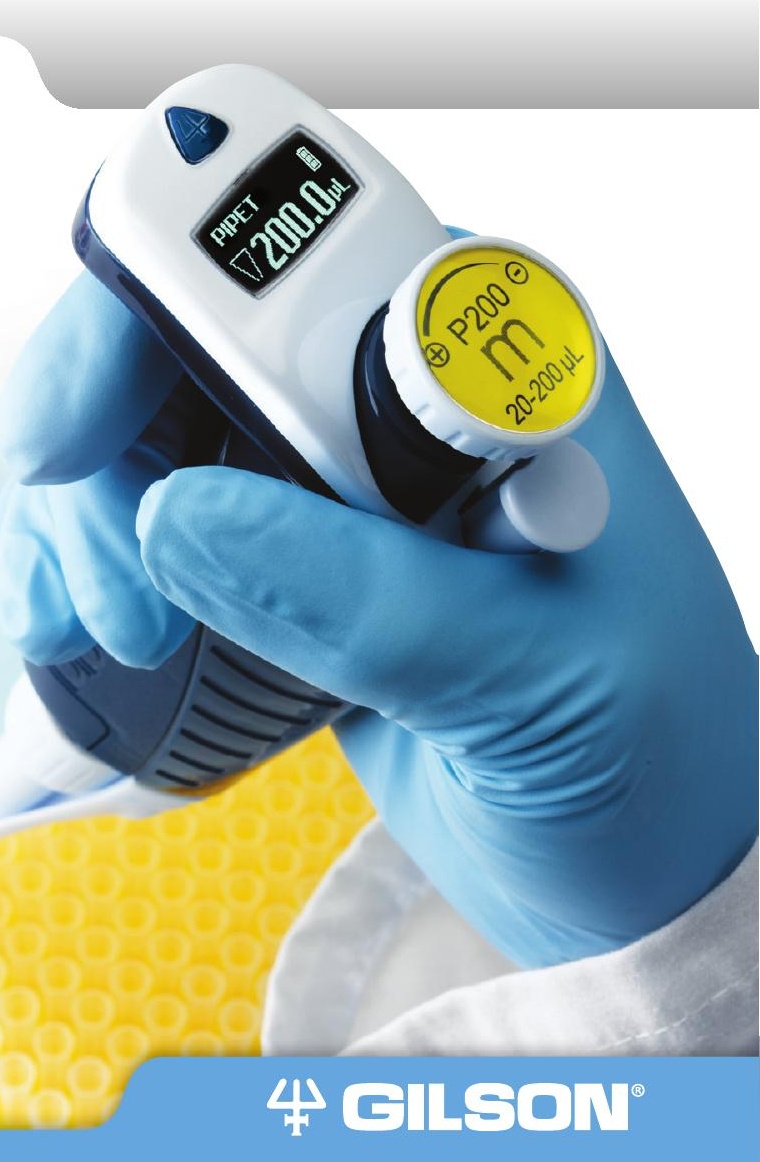


A

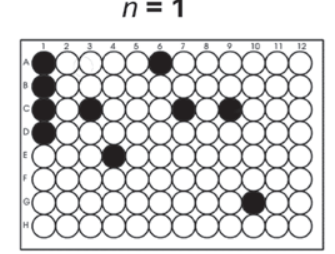

B

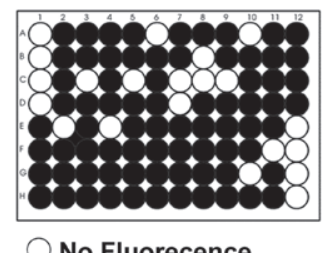

$\bigcirc$ No Fluorecence

$\Delta$ Fluorescence

C

D
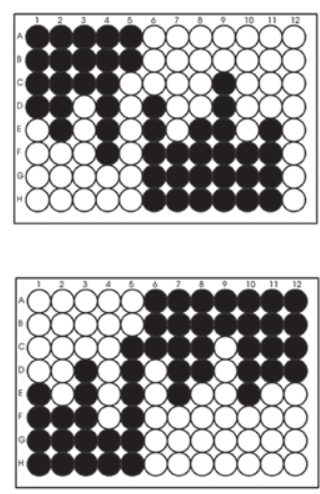

$n=2$
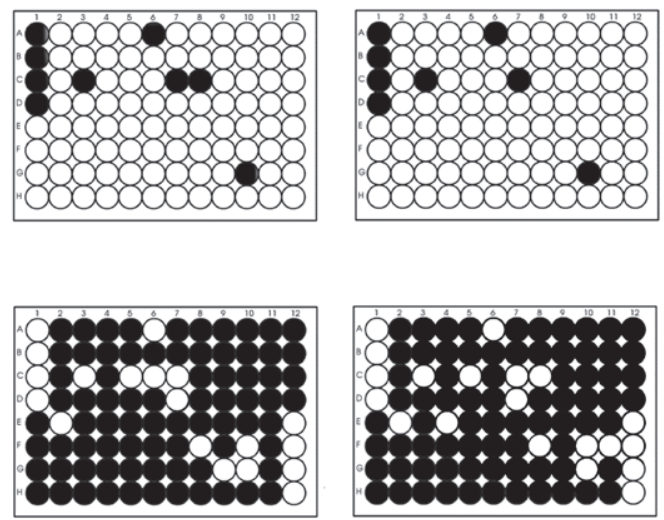

1
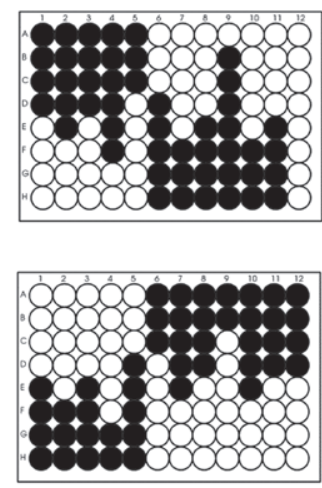
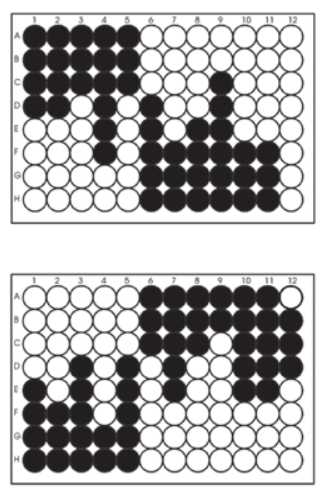

COMPOSITE

(AGONIST HITS)

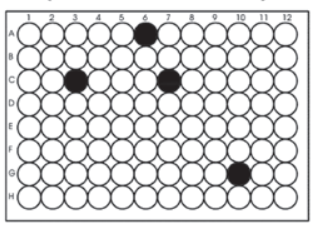

COMPOSITE

(ANTAGONIST HITS)

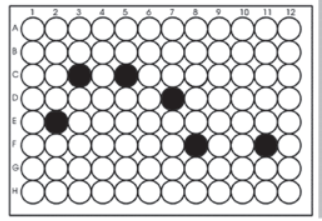

FINAL RESULT: Concentration-Response

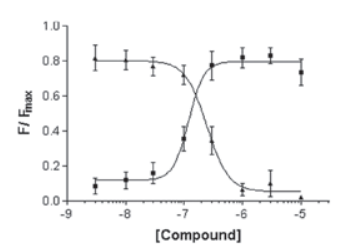

Concentration-Inhibition

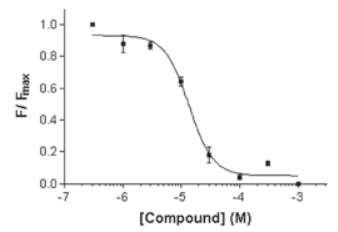

Figure 2. Method summary for the fluorometric screen. The method utilizes cells that stably express a receptor of interest. A different compound is added to each of the wells, and activation of receptors is measured as an increase in fluorescence (A). The same cells are then exposed to a second application consisting of a known agonist (B). Experiments were performed in triplicate, and compounds were considered hits when they elicit a response in two of the three plates (Composite, right-hand side of panels $A$ and $B$ ). We found that three replicates yielded the best returns (see Figure $3 \mathrm{~A}$ for more details). To get an accurate measure of the potency of the hits, the same method design was used using a serial dilution of the fragments; allowing concentration-response curves to be constructed from triplicate experiments ( $C$ and $D)$. Antagonists yielded standard concentration-inhibition curves, and agonist displayed a concentration-response relationship that was reversed in the second application because of receptor desensitization; the two curves crossed, acting as a further control.

(Equation 2). When affinity estimates from the two methods were compared, 44 of the 72 fragments (61\%) had $\mathrm{p} K_{\mathrm{i}}$ and $\mathrm{p} K_{\mathrm{b}}$ values with $<10$-fold difference, and several of these hits had affinities in the nanomolar range (Figure $3 \mathrm{~B}$ ). Of the remaining fragments, a further 19 had $<100$-fold difference. The majority of the compounds with $>10$-fold difference were at compound concentrations of $\geq 100 \mu \mathrm{M}$, a concentration at which it is technically difficult to get reliable affinity estimates using radioligand competition.

The tested fragments (1116) were derived from a larger proprietary compound collection $(>14,000)$ using a set of $1-D$ descriptors, such as molecular weight, $\log \mathrm{P}$, number of hydrogen bond donor/ acceptors, and rotatable bonds as selection criteria. An analysis of these fragments revealed a maximal structural diversity that was also present within the group of final hits. This was illustrated by the SCA as developed by Jun Xu (20). A complexity plot showed the high structural diversity of both agonists and antagonists, as hits were found throughout scaffold space (Figure 4). It should be noted that antagonist hits extended toward the higher complexity and lower cyclicity values, which can be explained by the higher molecular weight and bigger size of such fragments.

The affinities of known agonists [5-HT, m-chlorophenylbiguanide ( $m \mathrm{CPBG})]$ and competitive antagonists (granisetron, quinine, $d$-tubocurarine) were measured using the fluorometric assay and radioligand binding. Both techniques yielded similar affinities (all < 10-fold different) and were close to published values (Figure 3B) $(25,26)$. Of the 1044 fragments classified as inactive in the fluorometric assay, 20 were subjected to further analysis in order to ensure that potential candidates were not being missed. No detectable competition with radiolabeled granisetron was seen for 18 of these fragments, and for the remaining 2 fragments, competition with radioligand was at high concentrations $\left(K_{\mathrm{i}}>100 \mu \mathrm{M}\right)$. Thus, the incidence of missing positive hits was negligible $(21,22)$.

In summary, we describe a method design that utilizes a fluorescent microplate assay 
A

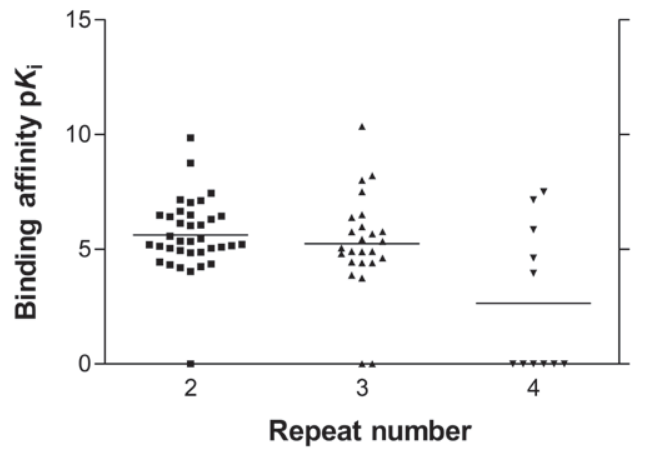

B

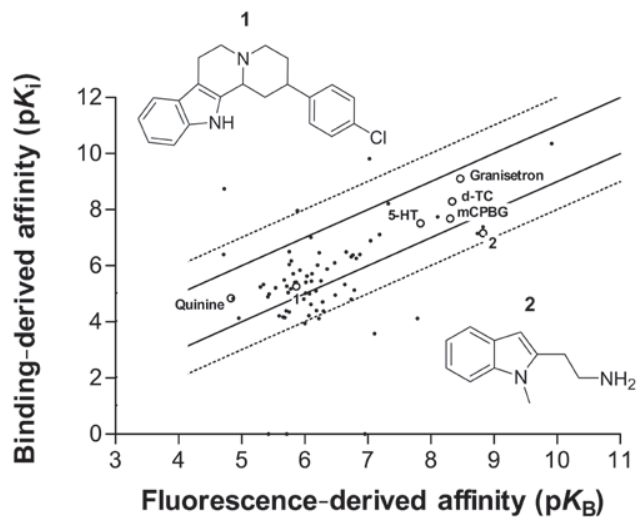

Figure 3. Parameters derived from the screen. (A). Optimizing the number of repeats needed to correctly identify agonists and antagonists. Compounds are categorized as hits when they exceeded a defined threshold (described in the "Categorizing agonists, antagonists, and non-hits" subsection) in at least two repeats of the same screen. In the graph, affinities (measured using radioligand) are plotted against the experimental repeat in which the fragment was first scored as a hit. Each point represents a different compound. The average affinity of the compounds in each repeat is shown as a horizontal bar. As the number of repeats was increased, the number of new hits declined (reduced number of points), the average affinity of the new hits decreased (horizontal line drops), and the incidence of false positives grew (increased number of points on the $x$ axis). (B) Correlation between affinities measured using the fluorescence-based assay and competition binding with $\left[{ }^{3} \mathrm{H}\right]$ granisetron. The two solid lines represent values $10 \times$ higher and lower than unity, and the dashed line is $100 \times$ higher and lower than unity. It has been assumed that all ligands were competitive, and no correction has been made for the fluorescence assay identifying ligands with noncompetitive or mixed competitive/noncompetitive activities. A selection of known 5-HT 3 receptor ligands were tested and are labeled, as are the novel fragments 1 and 2 . All affinities estimated from fluorescence and radioligand competition are calculated from at least three separate experiments.

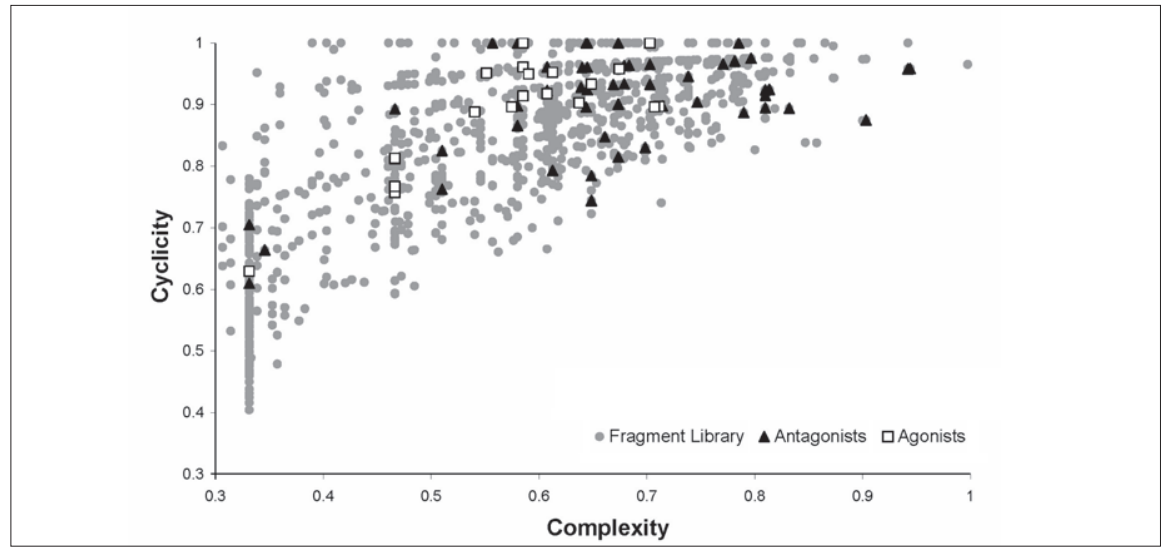

Figure 4. A scaffold-based classification approach (SCA) illustrates the high structural diversity of the identified hits. Cyclicity is a measure for the distribution of cyclic and acyclic parts of the structure, and complexity is a measure for size and shape of the scaffold.

for screening potential agonist and antagonists from a fragment-based compound library. The method is robust, sensitive, flexible, scalable, and cost-effective. The use of functional receptors in a mammalian cell system makes the technique physiologically relevant, and an extensive range of internal controls establishes the integrity of the results. The method requires no a priori decisions regarding the agonist or antagonist properties of the compound, since it identifies both in a single assay and it is independent of where the compounds bind on the receptor. The stringency of the assay can be altered by changing the concentrations of compound used in the initial screen or by altering the levels of fluorescence that are required to classify the compound as a hit. Because agonist and antagonist activity are assessed using the same plate of cells, cost and time savings are possible. Additionally, since both the initial screen and concentrationresponse assays are similarly designed, assay maintenance is simple. The method design can be applied to any ion channellinked response, including those mediated by LGICs, VGICs, or GPCRs.

\section{Acknowledgments}

We would like to thank the EU Neurocypres Project (FP7 grant to I.J.P.D.E. and S.C.R.L.) and the Wellcome Trust (to
S.C.R.L. and A.J.T.) for funding. S.C.R.L. is a Wellcome Trust Senior Research Fellow in Basic Biomedical Studies.

\section{Competing interests}

The authors declare no competing interests.

\section{References}

1. Hajduk, P.J. and J. Greer. 2007. A decade of fragment-based drug design: strategic advances and lessons learned. Nat. Rev. Drug Discov. 6:211-219.

2. Warr, W.A. 2009. Fragment-based drug discovery. J. Comput. Aided Mol. Des. 23:453458.

3. Congreve, M., G. Chessari, D. Tisi, and A.J. Woodhead. 2008. Recent developments in fragment-based drug discovery. J. Med. Chem. 51:3661-3680.

4. de Kloe, G.E., D. Bailey, R. Leurs, and I.J. de Esch. 2009. Transforming fragments into candidates: small becomes big in medicinal chemistry. Drug Discov. Today 14:630-646.

5. Chen, I.-J. and R.E. Hubbard. 2009. Lessons for fragment library design: analysis of output from multiple screening campaigns. J. Comput. Aided Mol. Des. 23:603-620.

6. Hajduk, P.J. 2006. Fragment-based drug design: how big is too big? J. Med. Chem. 49:6972-6976.

7. Wood, W.J., A.W. Patterson, H. Tsuruoka, R.K. Jain, and J.A. Ellman. 2005. Substrate activity screening: a fragment-based method for the rapid identification of nonpeptidic protease inhibitors. J. Am. Chem. Soc. 127:15521-15527.

8. Shuker, S.B., P.J. Hajduk, R.P. Meadows, and S.W. Fesik. 1996. Discovering high-affinity 
ligands for proteins: SAR by NMR. Science 274:1531-1534.

9. Smits, R.A., H.D. Lim, A. Hanzer, O.P. Zuiderveld, E. Guaita, M. Adami, G. Coruzzi, R. Leurs, and I.J. de Esch. 2008. Fragment based design of new $\mathrm{H} 4$ receptor-ligands with anti-inflammatory properties in vivo. J. Med. Chem. 51:2457-2467.

10. Ulens, C., A. Akdemir, A. Jongejan, R. van Elk, S. Bertrand, A. Perrakis, R. Leurs, A.B. Smit, et al. 2009. Use of acetylcholine binding protein in the search for novel alpha7 nicotinic receptor ligands. in silico docking, pharmacological screening, and X-ray analysis. J. Med. Chem. 52:2372-2383.

11. Thompson, A.J. and S.C.R. Lummis. 2007. The $5-\mathrm{HT}_{3}$ receptor as a therapeutic target. Expert Opin. Ther. Targets 11:527-540.

12. Webb, T.I. and J.W. Lynch. 2007. Molecular pharmacology of the glycine receptor chloride channel. Curr. Pharm. Des. 13:2350-2367.

13. Johnston, G.A. 2005. GABA receptor channe pharmacology. Curr. Pharm. Des. 11:1867-1885.

14. Olsen, R.W. and W. Sieghart. 2009. GABA receptors: subtypes provide diversity of function and pharmacology. Neuropharmacology 56:141-148.

15. Tang, W., J. Kang, X. Wu, D. Rampe, L. Wang, H. Shen, Z. Li, D. Dunnington, and T. Garyantes. 2001. Development and evaluation of high throughput functional assay methods for HERG potassium channel. J. Biomol. Screen. 6:325-331.
16. Shapiro, A.B., G.K. Walkup, and T.A. Keating. 2009. Correction for interference by test samples in high-throughput assays. J. Biomol. Screen. 14:1008-1016.

17. Leff, P. and I.G. Dougall. 1993. Further concerns over Cheng-Prusoff analysis. Trends Pharmacol. Sci. 14:110-112.

18. Cheng, Y. and W.H. Prusoff. 1973. Relationship between the inhibition constant (K1) and the concentration of inhibitor which causes 50 per cent inhibition (I50) of an enzymatic reaction. Biochem. Pharmacol. 22:3099-3108.

19. Siegal, G., E. Ab, and J. Schultz. 2007. Integration of fragment screening and library design. Drug Discov. Today 12:1032-1039.

20.Xu, J. 2002. A new approach to finding natural chemical structure classes. J. Med. Chem. 45:5311-5320

21. Hulme, E.C. and M.A. Trevethick. 2010. Ligand binding assays at equilibrium: validation and interpretation. Br.J. Pharmacol. Feb 2. [Epub ahead of print].

22.Gupta, A., A.K. Gupta, and K. Seshadri. 2009. Structural models in the assessment of protein druggability based on HTS data. J. Comput. Aided Mol. Des. 23:583-592.

23. Davies, D.R., B. Mamat, O.T. Magnusson, J. Christensen, M.H. Haraldsson, R. Mishra, B. Pease, E. Hansen, et al. 2009. Discovery of leukotriene A4 hydrolase inhibitors using metabolomics biased fragment crystallography. J. Med. Chem. 52:4694-4715.
24.Albert, J.S., N. Blomberg, A.L. Breeze, A.J. Brown, J.N. Burrows, P.D. Edwards, R.H. Folmer, S. Geschwindner, et al. 2007. An integrated approach to fragment-based lead generation: philosophy, strategy and case studies from AstraZeneca's drug discovery programmes. Curr. Top. Med. Chem. 7:16001629

25. Thompson, A.J. and S.C. Lummis. 2008 Antimalarial drugs inhibit human 5-HT and $\mathrm{GABA}_{\mathrm{A}}$ but not $\mathrm{GABA}_{\mathrm{C}}$ receptors. Br. J. Pharmacol. 153:1686-1696.

26. Brady, C.A., I.M. Stanford, I. Ali, L. Lin, J.M. Williams, A.E. Dubin, A.G. Hope, and N.M. Barnes. 2001. Pharmacological comparison of human homomeric $5-\mathrm{HT}_{3} \mathrm{~A}$ receptors versus heteromeric $5-\mathrm{HT}_{3} \mathrm{~A} /{ }_{3} \mathrm{~B}$ receptors. Neuropharmacology 41:282-284.

Received 24 May 2010; accepted 7 September 2010

Address correspondence to S.C.R. Lummis, Department of Biochemistry, University of Cambridge, Cambridge CB2 1QW, UK. e-mail: sl120@cam.ac.uk

To purchase reprints of this article, contact: carmelitag@fosterprinting.com

\section{We Illuminate Vour Life Science Researchl}

Life science and laboratory research professionals depend on high-quality Spectroline ${ }^{\circledR}$ ultraviolet products to make their work faster and easier. Our products are built to exacting standards, ensuring years of reliable, trouble-free service.

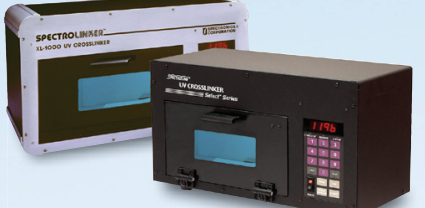

UV Crosslinkers

Provide accurate, error-free and super-fast membrane crosslinking and sample UV dosing

MiniMAX ${ }^{\mathrm{TM}}$ UV Viewing Station Space-saving mini-cabinet and UV lamp combination makes the perfect portable UV viewing station. Light weight and compact.

\section{UV-Protective Eye and Face Wear Specially designed to protect user against unwanted UV exposure.}

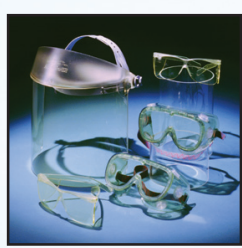

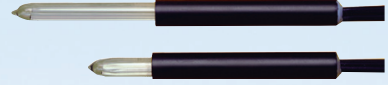

Miniature UV Pencil Lamps Double-bore quartz lamps used for wavelength calibration and emission. Custom lamps for OEM applications also available.
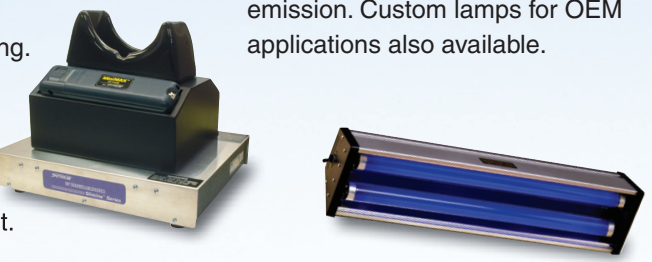

UV Bench and Display Lamps Lamps with various wattages and UV wavelengths provide both high intensity and wide area of light coverage.

Buy Direct From Us and Save!

Call 1-800-274-8888 (516-333-4840 outside USA) or email us at sales@spectroline.com and mention Code B510

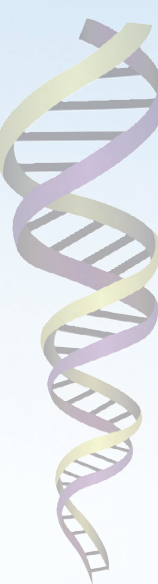
Advanced microprocessor-controlled units with software-driven functions. Ensure maximum accuracy and reliability in measurement of UV and visible light sources.

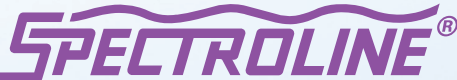

The World Leader in UV Technology! 